\title{
Eruptive xanthomas as a marker for metabolic disorders: A specific form of xanthoma that reflects hypertriglyceridemia
}

\author{
Souichiro Otaki ${ }^{1}$, Kenji Ashida ${ }^{1}$, Yuko Matsuo ${ }^{1}$, Kanoko Moritaka ${ }^{1}$, Shimpei Iwata ${ }^{1}$, Ayako \\ Nagayama $^{1}$, Aya Kawaguchi ${ }^{1}$, Hiroshi Koga ${ }^{1}$, Satoko Yoshinobu ${ }^{1}$, Nao Hasuzawa ${ }^{1}$, Seiichi \\ Motomura $^{1}$, Jun Akiba ${ }^{1}$, Masatoshi Nomura ${ }^{1}$, and Takekuni Nakama ${ }^{1}$ \\ ${ }^{1}$ Kurume University School of Medicine
}

February 8, 2022

\begin{abstract}
Eruptive xanthomas are skin manifestations associated with hypertriglyceridemia. Accordingly, improvement of hypertriglyceridemia can ameliorate this condition. We report a case of a patient with type 2 diabetes mellitus who was diagnosed with this skin lesion. Clinicians should be aware that eruptive xanthomas could indicate metabolic disorders associated with atherosclerosis.
\end{abstract}

Eruptive xanthomas as a marker for metabolic disorders: A specific form of xanthoma that reflects hypertriglyceridemia

\section{Authors:}

Souichiro Otaki ${ }^{1}$, Kenji Ashida ${ }^{*}$, Yuko Matsuo ${ }^{1}$, Kanoko Moritaka $^{1}$, Shimpei Iwata ${ }^{1}$, Ayako Nagayama ${ }^{1}$, Aya Kawaguchi $^{2,3}$, Hiroshi Koga ${ }^{3}$, Satoko Yoshinobu ${ }^{1}$, Nao Hasuzawa ${ }^{1}$, Seiichi Motomura ${ }^{1}$, Jun Akiba ${ }^{2}$, Takekuni Nakama ${ }^{3}$, Masatoshi Nomura ${ }^{1}$

\section{Affiliations:}

${ }^{1}$ Division of Endocrinology and Metabolism, Department of Internal Medicine, Kurume University School of Medicine, Fukuoka, Japan

${ }^{2}$ Department of Diagnostic Pathology, Kurume University Hospital, Fukuoka, Japan

${ }^{3}$ Department of Dermatology, Kurume University School of Medicine, Fukuoka, Japan

*Correspondence:

Kenji Ashida, MD, PhD

Division of Endocrinology and Metabolism, Department of Internal Medicine, Kurume University School of Medicine, 67 Asahi-machi, Kurume-city, Fukuoka, 830-0011 Japan

Phone: +81-942-31-7563

Fax: +81-942-35-8943

E-mail: ashida@med.kurume-u.ac.jp

Key Clinical Message: 
Eruptive xanthomas are associated with hypertriglyceridemia due to dysregulated lipid and glucose metabolism. Clinicians should not overlook eruptive xanthomas as possible indicators of metabolic disorders associated with cardiovascular disease.

\begin{abstract}
Eruptive xanthomas are skin manifestations associated with hypertriglyceridemia. Accordingly, improvement of hypertriglyceridemia can ameliorate this condition. We report a case of a patient with type 2 diabetes mellitus who was diagnosed with this skin lesion. Clinicians should be aware that eruptive xanthomas could indicate metabolic disorders associated with atherosclerosis.
\end{abstract}

\title{
Key words:
}

Eruptive xanthomas; Hypertriglyceridemia; Diabetes mellitus; Insulin action failure; Atherosclerosis.

\section{E-mail address of each author:}

Souichiro Otaki: ohtaki_souichirou@med.kurume-u.ac.jp

Kenji Ashida: ashida@med.kurume-u.ac.jp

Yuko Matsuo: matsuo_yuuko@med.kurume-u.ac.jp

Kanoko Moritaka: moritaka_kanoko@med.kurume-u.ac.jp

Shimpei Iwata: iwata_shinpei@med.kurume-u.ac.jp

Ayako Nagayama: nagayama_ayako@med.kurume-u.ac.jp

Aya Kawaguchi: nanri_aya@med.kurume-u.ac.jp

Hiroshi Koga: hiroshi_koga@kurume-u.ac.jp

Satoko Yoshinobu: yoshinobu_satoko@med.kurume-u.ac.jp

Nao Hasuzawa: hasuzawa@med.kurume-u.ac.jp

Seiichi Motomura: motomura_seiichi@kurume-u.ac.jp

Jun Akiba: akiba@med.kurume-u.ac.jp

Takekuni Nakama: takekuni@med.kurume-u.ac.jp

Masatoshi Nomura: nomura@med.kurume-u.ac.jp

\section{1 | INTRODUCTION}

Early diagnosis and treatment of dyslipidemia are required to prevent the progression of atherosclerosis. Hypertriglyceridemia has been shown to increase the risk of atherosclerosis, which can lead to the development of cardiovascular disease. ${ }^{1}$ Diabetes mellitus is frequently associated with dyslipidemia, particularly type IIb, III, or IV hyperlipidemias, which can promote hypertriglyceridemia. ${ }^{2,3}$

Xanthomas are typical skin lesions associated with dyslipidemia that occur as accumulations especially in the Achilles and patellar tendons, extensor tendons of the hands and elbows, eyelids, trunk, and buttocks. ${ }^{4}$ These skin lesions have been associated with marked hypercholesterolemia, which is typically observed in patients with familial hyperlipidemia type IIa. ${ }^{5}$

With regard to hypertriglyceridemia, evidence suggests that eruptive xanthomas were associated with serum triglyceride levels. ${ }^{6}$ Considering the availability of studies on the clinical courses of eruptive xanthoma,${ }^{7-9}$ clinicians should familiarize themselves with the details of this condition for correct diagnosis in the early stage. 
We herein report on a patient with dyslipidemia complicated with type 2 diabetes mellitus, in whom eruptive xanthoma served as an indicator for hypertriglyceridemia. After improvement of hypertriglyceridemia through diet and medical treatment, the color tones of the skin lesions had changed from red to white, ultimately disappearing after several months.

\section{2 | CASE HISTORY/EXAMINATION}

A 35-year-old Chinese man was admitted to Kurume University Hospital on May, 2020 owing to fatigue and hyperglycemia. He stated that hyperlipidemia and hyperglycemia started 2 years prior but that he discontinued any treatment. He had neither any familial history of dyslipidemia and diabetes mellitus nor a life history of drug, alcohol, or smoking abuse. On examination, his body mass index and abdominal circumference were $32 \mathrm{~kg} / \mathrm{m}^{2}$ and $106.1 \mathrm{~cm}$, respectively. Multiple clustered papules were observed on the bilateral extremities (Figure 1). Histopathological examinations of skin biopsy specimens led to the diagnosis of eruptive xanthomas (Figure 2). Laboratory examinations demonstrated high levels of fasting serum triglyceride $(1871 \mathrm{mg} / \mathrm{dL})$ and total cholesterol $(371 \mathrm{mg} / \mathrm{dL})$ and low levels of high-density lipoprotein cholesterol (22 mg/dL). However, low-density lipoprotein (LDL) cholesterol levels were within the normal range. Examination of lipoprotein fraction showed that the mid-band and small-dense LDL was contained (Figure 3). Chronic hyperglycemia was also denoted, with a fasting plasma glucose level of $203 \mathrm{mg} / \mathrm{dL}$ and HbA1c value (NGSP) of $9.9 \%$ (Table 1). Other examinations, including electrocardiogram and chest radiograph, were unremarkable. Ultrasonography revealed moderate to severe fatty liver (Figure 4). Diet therapy with $1600 \mathrm{kcal} /$ day calorie restriction and $0.2 \mathrm{mg} /$ day of pemafibrate, which was ultimately increased to 0.4 $\mathrm{mg} /$ day orally, were initiated to reduce serum lipids. Additionally, $500 \mathrm{mg} /$ day of metformin and $10 \mathrm{mg} /$ day of empagliflozin were administrated to improve insulin sensitivity and hyperglycemia. Finally, both serum triglyceride and plasma glucose levels improved to 425 and $101 \mathrm{mg} / \mathrm{dL}$, respectively, with a concomitant change in color of the skin lesions from red to white and a decrease in the number of eruptions until total eradication.

\section{3 | DISCUSSION}

Eruptive xanthomas develop along with marked hypertriglyceridemia and are an important indicator of metabolic disorders, including dyslipidemia and diabetes mellitus. Grouped papular eruptions 1-4 mm in diameter are specifically observed in the skin over the buttocks, posterior portion of the thigh, elbows, and lumbar region. ${ }^{10,11}$ Accumulation of foaming cells derived from macrophage phagocytosis of remnant lipoprotein is observed on histopathological examination. ${ }^{11,}{ }^{12}$ Hypertriglyceridemia is the highest risk factor for the development of eruptive xanthomas, with $8.5 \%$ of the patients with hypertriglyceridemia above 20 $\mathrm{mmol} / \mathrm{L}(1772 \mathrm{mg} / \mathrm{dL})$ developing this condition and subsequently improving after a reduction in serum triglyceride level. ${ }^{8,13}$ In this context, hypertriglyceridemia and diabetes mellitus have been considered major causative factors for eruptive xanthoma and need to be treated to prevent the progressions of systemic atherosclerosis. ${ }^{14}$ Clinicians should be aware that this type of skin lesions can indicate the presence of metabolic disorders, which need to be addressed in order to improve the eruptions ${ }^{11}$ and prevent cardiovascular events. $^{14}$

Insulin insufficiency is a major factor for increased remnant lipoprotein, including chylomicron or very lowdensity lipoprotein (VLDL), and the manifestation of xanthoma and systemic atherosclerosis. A putative relationship between eruptive xanthoma and atherosclerosis is summarized in Figure 5. Obesity and diabetes mellitus can promote insulin insufficiency in extra adipose tissues due to insulin insensitivity caused by the following factors: (1) lipotoxicity, increased skeletal muscle triglyceride content followed by elevation of free fatty acids ${ }^{15}$; (2) changes in adipokines, including low adiponectin ${ }^{16}$ and high leptin ${ }^{17}$ levels; (3) elevations in proinflammatory cytokines, including tumor necrosis factor- $\alpha$, IL- $1 \beta$, and IL- 6 levels, in the adipose tissues ${ }^{18}$; (4) activation of the endoplasmic reticulum and related signaling networks ${ }^{19}$; and (5) elevated hexosamine flux in adipose tissues. ${ }^{20}$ Insulin insufficiency decreases lipoprotein lipase activity by activating angiopoietinlike protein 3 (ANGPTL3) ${ }^{21-23}$ expressed in the liver, which increases the levels of VLDL and triglyceride via suppression of lipoprotein lipase activity ${ }^{24}$ and overproduction through lipolysis-derived free fatty acids and glycerol. ${ }^{23}$. Thus, high ANGPLT3 activity in patients with hyperglycemia or obesity can induce elevations of 
serum remnant lipoproteins, chylomicron, or LDL, and VLDL levels. ${ }^{24}$ Remnants infiltrating into the vessel walls or skin ${ }^{25}$ are recognized and engulfed by macrophages. After phagocytosis, macrophages change to foam cells and are deposited into the vessel walls and skin, ${ }^{11}$ which lead to arthrosclerosis ${ }^{26}$ and eruptive xanthoma, ${ }^{12}$ respectively.

Hypertriglyceridemia should be treated early to prevent progression to acute pancreatitis and cardiovascular events. Severe hypertriglyceridemia over $1000 \mathrm{mg} / \mathrm{dL}$ has been found to markedly increase the risk of developing acute pancreatitis. ${ }^{27}$ Postprandial hypertriglyceridemia is positively associated with the development of ischemic heart disease, myocardial infarction, and cardiovascular events independent of serum cholesterol levels. ${ }^{28}$ In this context, casual hypertriglyceridemia, including postprandial levels as high as fasting levels, have also been indicated to significantly increase the risks of developing cardiovascular events. ${ }^{29}$ Additionally, triglyceride-rich lipoprotein and remnant apo-B48-positive chylomicron derived from short intestine are increased during hypertriglyceridemia. ${ }^{30}$ Patients with high fasting levels of apo-lipoprotein B48 have been found to be at significant risk for developing coronary artery stenosis. ${ }^{31,32}$ Thus, hypertriglyceridemia requires interventions to prevent the progression of cardiovascular diseases and improve prognosis.

In conclusion, this report details our experience with a patient who presented with hypertriglyceridemia and type 2 diabetes mellitus concurrent with eruptive xanthoma, which was ameliorated by the treatment of dyslipidemia and hyperglycemia. Eruptive xanthoma can help clinicians determine the presence of hypertriglyceridemia and insulin insensitivity induced by obesity and diabetes mellitus, as well as genetic disorders related with lipoprotein metabolism. Clinicians should therefore be aware of skin manifestations of metabolic disorders, which can lead to atherosclerosis.

\section{ACKNOWLEDGMENTS}

This research received no specific grant from any funding agency in the public, commercial, or not-for-profit sectors. The authors would like to thank Enago (www.enago.jp) for the English language review.

\section{CONSENT}

All procedures complied with the ethical standards of the Institutional Review Board of the Kurume University School of Medicine and the 2013 Declaration of Helsinki. This report was approved by the Ethics Committee of Kurume University Hospital (2021-067). The patient provided written informed consent for publication of this study.

\section{AUTHOR CONTRIBUTIONS}

SO: study design, data collection, drafting, interpretation of data, and revision.

KA: study design, data collection, drafting, interpretation of the data, review, and revision.

YM: data collection, interpretation of the data, and review.

KM: data collection, interpretation of the data, and review.

SI: interpretation of the data, and review.

AN: interpretation of the data, and review.

AK: data collection, interpretation of the data, and review.

SM: interpretation of the data, and review.

JA: data collection, interpretation of the data, and review.

TN: data collection, interpretation of the data, and review.

MN: study design, drafting, interpretation of the data, review, and revision.

All authors provided inputs for preparation of the manuscript and have read and approved the final version for submission. 


\section{CONFLICT OF INTEREST}

The authors have no competing interests.

\section{REFERENCES}

1. Bhatt DL, Steg PG, Miller M, Brinton EA, Jacobson TA, Ketchum SB, Doyle RT Jr., Juliano RA, Jiao L, Granowitz C, Tardif JC, Ballantyne CM. Cardiovascular Risk Reduction with Icosapent Ethyl for Hypertriglyceridemia. N Engl J Med. 2019; 380 (1): 11-22. https://doi.org/10.1056/NEJMoa1812792.

2. Arai H, Ishibashi S, Bujo H, Hayashi T, Yokoyama S, Oikawa S, Kobayashi J, Shirai K, Ota T, Yamashita S, Gotoda T, Harada-Shiba M, Sone H, Eto M, Suzuki H, Yamada N. Management of type IIb dyslipidemia.J Atheroscler Thromb. 2012; 19 (2): 105-114. https://doi.org/10.5551/jat.10447.

3. Schaefer JR. Unraveling hyperlipidemia type III (dysbetalipoproteinemia), slowly. Eur J Hum Genet. 2009;17 (5): 541-542. https://doi.org/10.1038/ejhg.2008.222.

4. Maharaj S, Chang S, Nayak SB. Familial hypercholesterolemia presenting with multiple nodules of the hands and elbow. Clin Case Rep. 2015; 3 (6): 411-414. https://doi.org/10.1002/ccr3.249.

5. Harada-Shiba M, Arai H, Ishigaki Y, Ishibashi S, Okamura T, Ogura M, Dobashi K, Nohara A, Bujo H, Miyauchi K, Yamashita S, Yokote K. Guidelines for Diagnosis and Treatment of Familial Hypercholesterolemia 2017. J Atheroscler Thromb. 2018; 25 (8): 751-770. https://doi.org/10.5551/jat.CR003.

6. Roga G, Jithendriya M. Eruptive xanthoma: Warning sign of systemic disease. Cleve Clin J Med. 2016; 83 (10): 715-716. https://doi.org/10.3949/ccjm.83a.15126.

7. Solak B, Kara RO, Acikgoz SB, Kosem M. First and only symptom of undiagnosed diabetes mellitus: eruptive xanthoma. BMJ Case Rep.2015; 2015 : bcr2015212160. https://doi.org/10.1136/bcr-2015-212160.

8. Tsuchiya S, Sawada S, Takeda K, Takahashi K, Nakajima T, Kohata M, Kurosawa S, Satake C, Imai J, Kikuchi K, Aiba S, Katagiri H. Eruptive xanthomas in a patient with soft-drink diabetic ketosis and apolipoprotein E4/2. Endocr J. 2019; 66 (1): 107-114. https://doi.org/10.1507/endocrj.EJ18-0356.

9. Kashif M, Kumar H, Khaja M. An unusual presentation of eruptive xanthoma: A case report and literature review. Medicine (Baltimore). 2016; 95 (37): e4866. https://doi.org/10.1097/MD.0000000000004866.

10. Abdelghany M, Massoud S. Eruptive xanthoma. Cleve Clin J Med.2015; 82 (4): $209-210$. https://doi.org/10.3949/ccjm.82a.14081.

11. Zaremba J, Zaczkiewicz A, Placek W. Eruptive xanthomas.Postepy Dermatol Alergol. $2013 ; 30$ (6): 399-402. https://doi.org/10.5114/pdia.2013.39439.

12. Zak A, Zeman M, Slaby A, Vecka M. Xanthomas: clinical and pathophysiological relations. Biomed Pap Med Fac Univ Palacky Olomouc Czech Repub. 2014; 158 (2): 181-188. https://doi.org/10.5507/bp.2014.016.

13. Sandhu S, Al-Sarraf A, Taraboanta C, Frohlich J, Francis GA. Incidence of pancreatitis, secondary causes, and treatment of patients referred to a specialty lipid clinic with severe hypertriglyceridemia: a retrospective cohort study. Lipids Health Dis. 2011; 10 : 157. https://doi.org/10.1186/1476-511X-10-157.

14. Boullart ACI, de Graaf J, Stalenhoef AF. Serum triglycerides and risk of cardiovascular disease. Biochim Biophys Acta. 2012;1821 (5): 867-875. https://doi.org/10.1016/j.bbalip.2011.10.002.

15. Samuel VT, Shulman GI. Mechanisms for insulin resistance: common threads and missing links. Cell. 2012; 148(5): 852-871. https://doi.org/10.1016/j.cell.2012.02.017.

16. Yamauchi T, Kadowaki T. Adiponectin receptor as a key player in healthy longevity and obesity-related diseases. Cell Metab. 2013;17 (2): 185-196. https://doi.org/10.1016/j.cmet.2013.01.001.

17. Zhou Y, Rui L. Leptin signaling and leptin resistance. Front Med. 2013; 7 (2): $207-222$. https://doi.org/10.1007/s11684-013-0263-5. 
18. Grant RW, Dixit VD. Adipose tissue as an immunological organ. Obesity (Silver Spring). 2015; 23 (3): 512-518. https://doi.org/10.1002/oby.21003.

19. Hotamisligil GS. Endoplasmic reticulum stress and the inflammatory basis of metabolic disease. Cell. 2010; 140 (6): 900-917. https://doi.org/10.1016/j.cell.2010.02.034.

20. Teo CF, Wollaston-Hayden EE, Wells L. Hexosamine flux, the O-GlcNAc modification, and the development of insulin resistance in adipocytes. Mol Cell Endocrinol. 2010; 318 (1-2): 44-53. http://doi.org/10.1016/j.mce.2009.09.022.

21. Romeo S, Yin W, Kozlitina J, Pennacchio LA, Boerwinkle E, Hobbs HH, Cohen JC. Rare loss-of-function mutations in ANGPTL family members contribute to plasma triglyceride levels in humans. $J$ Clin Invest. 2009; 119 (1): 70-79. https://doi.org/10.1172/JCI37118.

22. Inukai K, Nakashima Y, Watanabe M, Kurihara S, Awata T, Katagiri H, Oka Y, Katayama S. ANGPTL3 is increased in both insulin-deficient and -resistant diabetic states. Biochem Biophys Res Commun. 2004;317 (4): 1075-1079. https://doi.org/10.1016/j.bbrc.2004.03.151.

23. Shimamura M, Matsuda M, Kobayashi S, Ando Y, Ono M, Koishi R, Furukawa H, Makishima M, Shimomura I. Angiopoietin-like protein 3, a hepatic secretory factor, activates lipolysis in adipocytes. Biochem Biophys Res Commun. 2003; 301 (2): 604-609. https://doi.org/10.1016/s0006-291x(02)03058-9.

24. Chen PY, Gao WY, Liou JW, Lin CY, Wu MJ, Yen JH. Angiopoietin-Like Protein 3 (ANGPTL3) Modulates Lipoprotein Metabolism and Dyslipidemia.Int J Mol Sci. 2021; 22 (14): 7310. https://doi.org/10.3390/ijms22147310.

25. Toth PP. Triglyceride-rich lipoproteins as a causal factor for cardiovascular disease. Vasc Health Risk Manag. 2016;12 : 171-183. https://doi.org/10.2147/VHRM.S104369. 26. Lusis AJ. Atherosclerosis. Nature. 2000. 14; 407(6801): 233-241. https://doi.org/10.1038/35025203.

27. Parhofer KG, Laufs U. The Diagnosis and Treatment of Hypertriglyceridemia. Dtsch Arztebl Int. 2019; 116 (49): 825-832. https://doi.org/10.3238/arztebl.2019.0825.

28. Iso H, Naito Y, Sato S, Kitamura A, Okamura T, Sankai T, Shimamoto T, Iida M, Komachi Y. Serum triglycerides and risk of coronary heart disease among Japanese men and women. Am J Epidemiol. 2001; 153 (5): 490-499. https://doi.org/10.1093/aje/153.5.490.

29. Marston NA, Giugliano RP, Im K, Silverman MG, O'Donoghue ML, Wiviott SD, Ference BA, Sabatine MS. Association Between Triglyceride Lowering and Reduction of Cardiovascular Risk Across Multiple Lipid-Lowering Therapeutic Classes: A Systematic Review and MetaRegression Analysis of Randomized Controlled Trials. Circulation. 2019;140 (16): 1308-1317. https://doi.org/10.1161/CIRCULATIONAHA.119.041998.

30. Masuda D, Yamashita S. Postprandial Hyperlipidemia and Remnant Lipoproteins. J Atheroscler Thromb. 2017; 24 (2): 95-109. https://doi.org/10.5551/jat.RV16003.

31. Alipour A, Valdivielso P, Elte JWF, Janssen HW, Rioja J, van der Meulen N, Mechelen R, Njo TL, González-Santos P, Rietveld AP, Cabezas MC. Exploring the value of apoB48 as a marker for atherosclerosis in clinical practice. Eur J Clin Invest. 2012; 42 (7): 702-708. https://doi.org/10.1111/j.13652362.2011.02635.x.

32. Masuda D, Sugimoto T, Tsujii K, Inagaki M, Nakatani K, Yuasa-Kawase M, Tsubakio-Yamamoto K, Ohama T, Nishida M, Ishigami M, Kawamoto T, Matsuyama A, Sakai N, Komuro I, Yamashita S. Correlation of fasting serum apolipoprotein B-48 with coronary artery disease prevalence.Eur J Clin Invest. 2012; 42 (9): 992-999. https://doi.org/10.1111/j.1365-2362.2012.02687.x.

Table 1. Laboratory data at admission

Parameters 
Table 1. Laboratory data at admission

Complete blood cell count

Red blood cell count, $\times 10^{4} / \mu \mathrm{L}$

White blood cell count, $/ \mu \mathrm{L}$

Platelet, $\times 10^{4} / \mu \mathrm{L}$

Endocrinology

Adrenocorticotropic hormone, $\mathrm{pg} / \mathrm{mL}$

Cortisol, $\mu \mathrm{g} / \mathrm{dL}$

Dehydroepiandrosterone-sulfate, $\mu \mathrm{g} / \mathrm{dL}$

Growth hormone, $\mathrm{ng} / \mathrm{mL}$

Insulin-like growth factor- $1, \mathrm{ng} / \mathrm{mL}$

Prolactin, ng $/ \mathrm{mL}$

Thyroid stimulating hormone, $\mu \mathrm{IU} / \mathrm{mL}$

Free thyroxine, $\mathrm{ng} / \mathrm{dL}$

Luteinizing hormone, $\mathrm{mIU} / \mathrm{mL}$

Follicular stimulating hormone, $\mathrm{mIU} / \mathrm{mL}$

Abbreviations: HbA1c, hemoglobin A1c; LDL-C, low-density lipoprotein cholesterol; NGSP, National Glycohemoglobin Sta

Figure legends:

Figure 1. Clinical images of eruptive xanthomas

Multiple red to salmon pink papule clusters. (a) Bilateral thigh, front. (b) Bilateral thigh, back. (c) Left forearm.

Figure 2. Histological findings of a skin biopsy specimen from the left forearm

Pathophysiological examination with hematoxylin eosin staining. (a) Appearance of eruptions. An eruption surrounded by four dotted markings was investigated using microscopy. (b) Low magnitude $(\times 40)$. Massive foam cells (indicated by arrows) infiltrating into the superficial layer of dermis. (c) High magnitude (×400). Eosinophilic substrate (asterisks), probably indicating extracellular lipids, were observed between collagen fibers. Additionally, foam cells are indicated by arrows.

Figure 3. Lipoprotein profiles

Lipoprotein fractions are demonstrated using polyacrylamide gel electrophoresis. (a) Results of lipoprotein fraction in wave expression method. (b) Results of polyacrylamide gel staining. Compared to control subjects.

Figure 4. Ultrasonography

Abdominal ultrasonography revealing a bright liver.

Figure 5. Summary of the pathological relationships between eruptive xanthoma, hypertriglyceridemia, and atherosclerosis

Obesity and diabetes mellitus have potentials to increase serum remnant lipoproteins, namely TG-rich lipoproteins, and lead to the hypertriglyceridemia. Marked increase in the levels of serum TG-rich remnant lipoproteins infiltrate into the subcutaneous tissue and would be taken up by migrated macrophages, which turn into lipid-filled foam cells. The accumulation of these foam cells increases the risks of developing eruptive xanthomas in the subcutaneous tissue or atherosclerosis in the vessels. 
Abbreviations: ANGPLT3: angiopoietin-like protein 3, FFA: free fatty acid, IL-1 $\beta$ : interleukin-1 $\beta$, IL-6: interleukin-6, LPL: lipoprotein lipase, TG: triglyceride, TNF- $\alpha$ : tumor necrosing factor- $\alpha$, VLDL: very lowdensity lipoprotein.

(a)

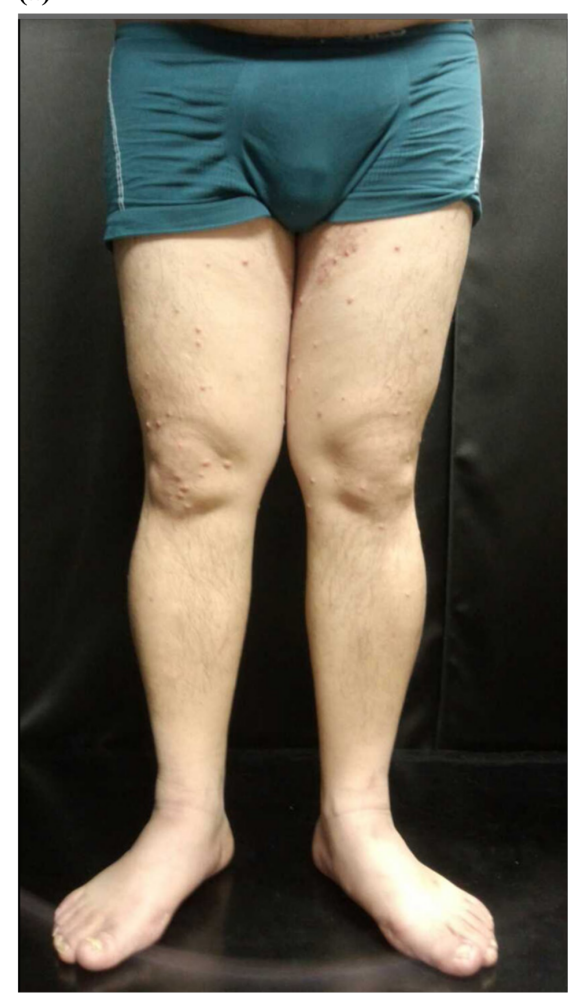

(c) (b)

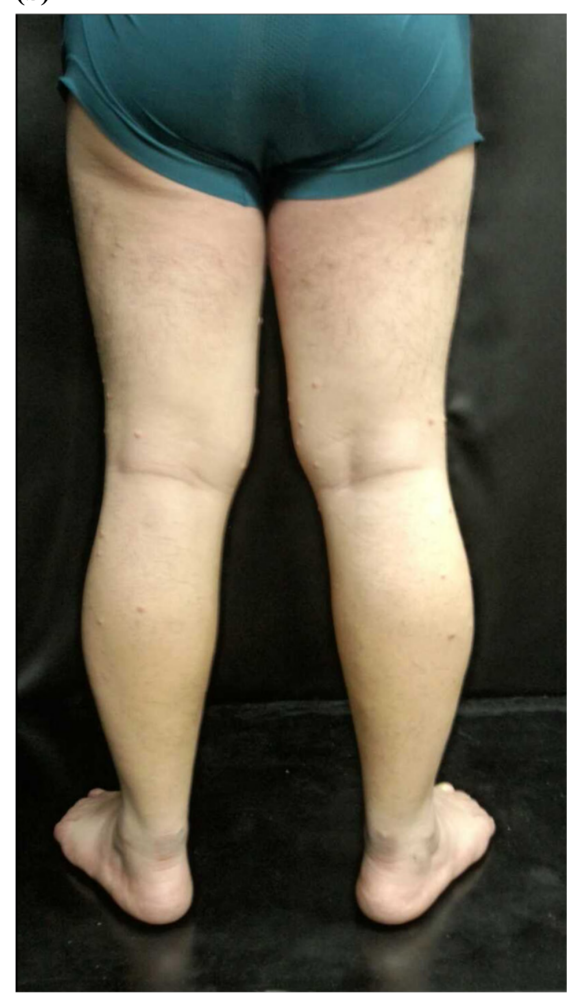

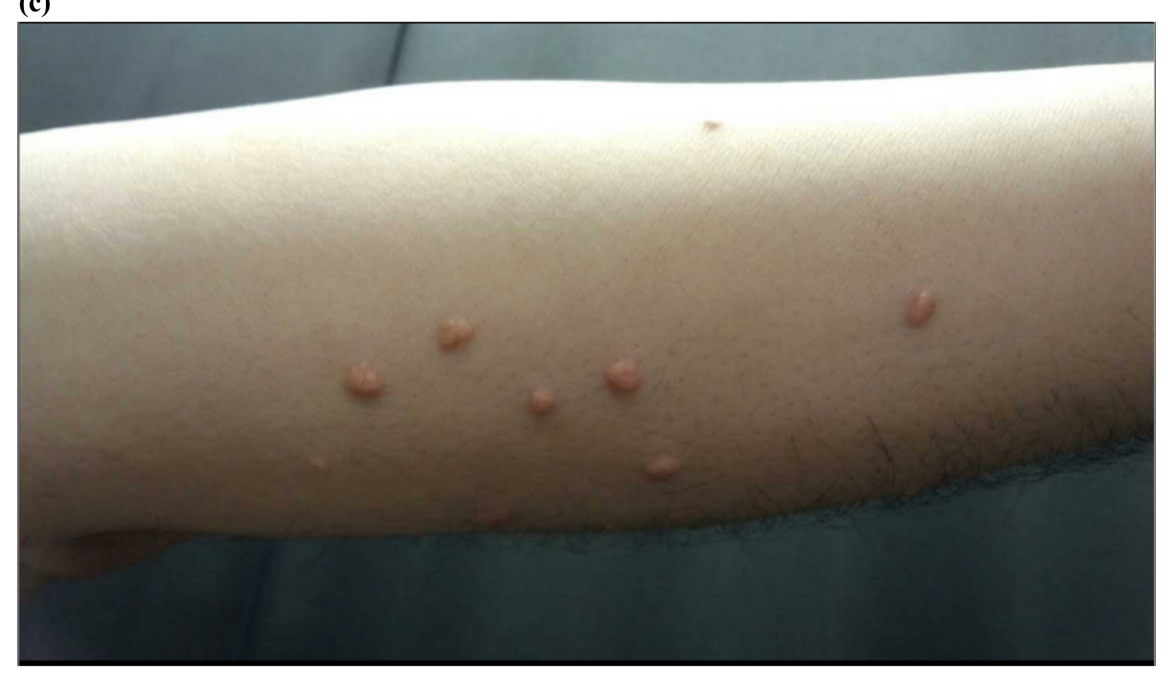

Figure 1 
(a)

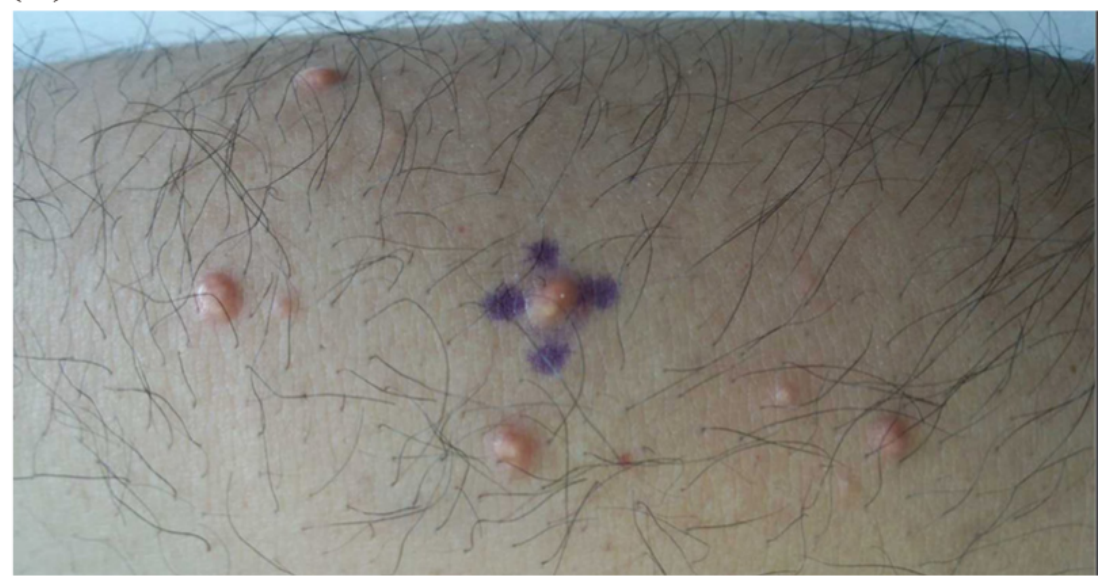

(b)

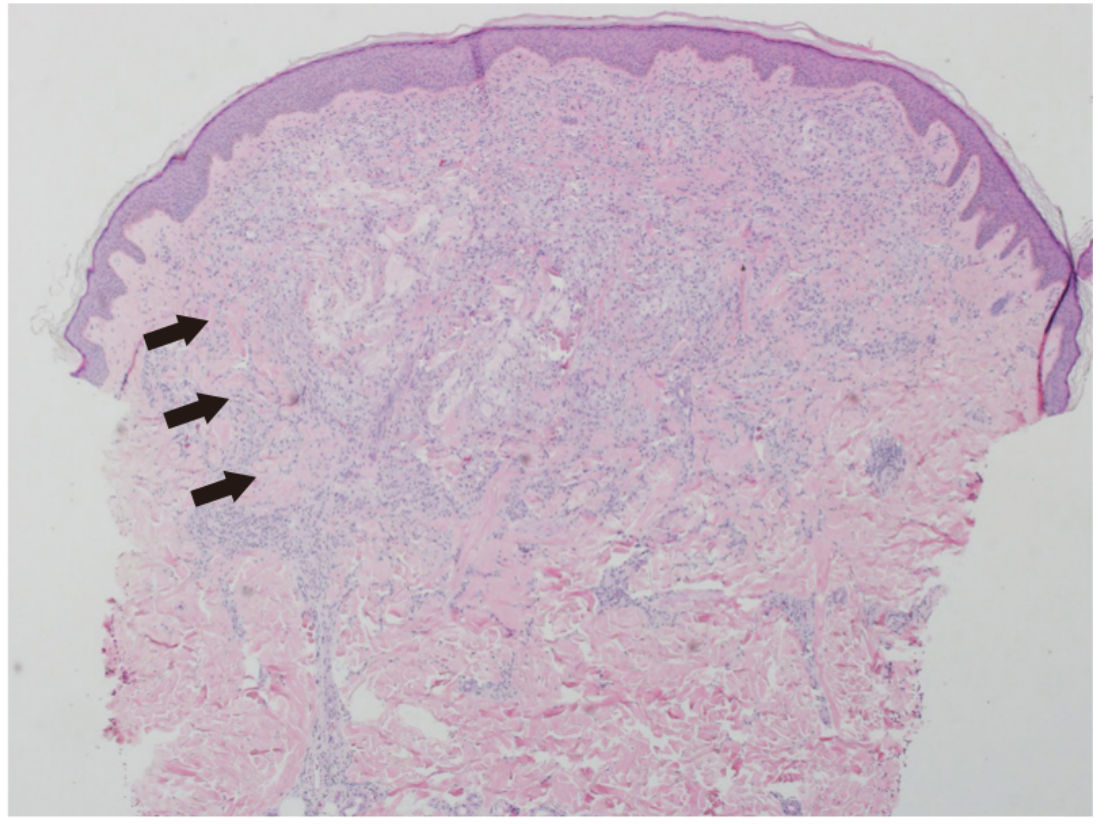

(c)

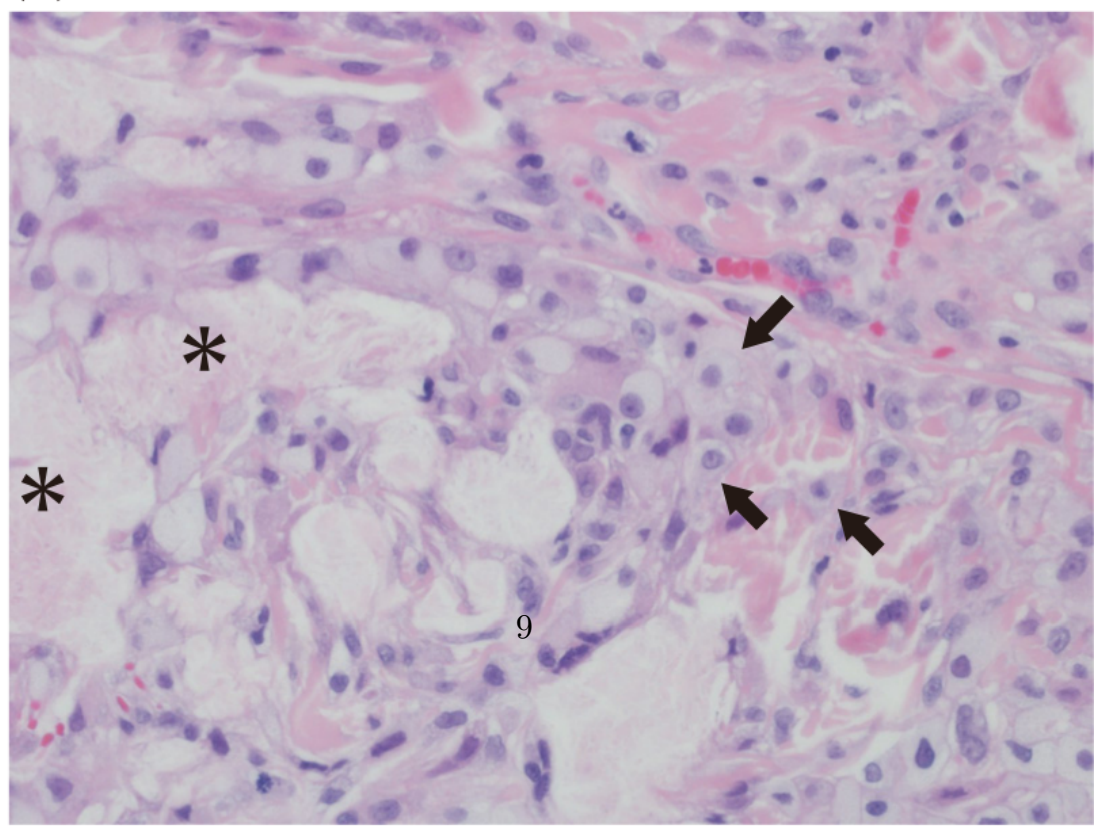

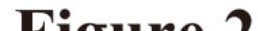


(a)

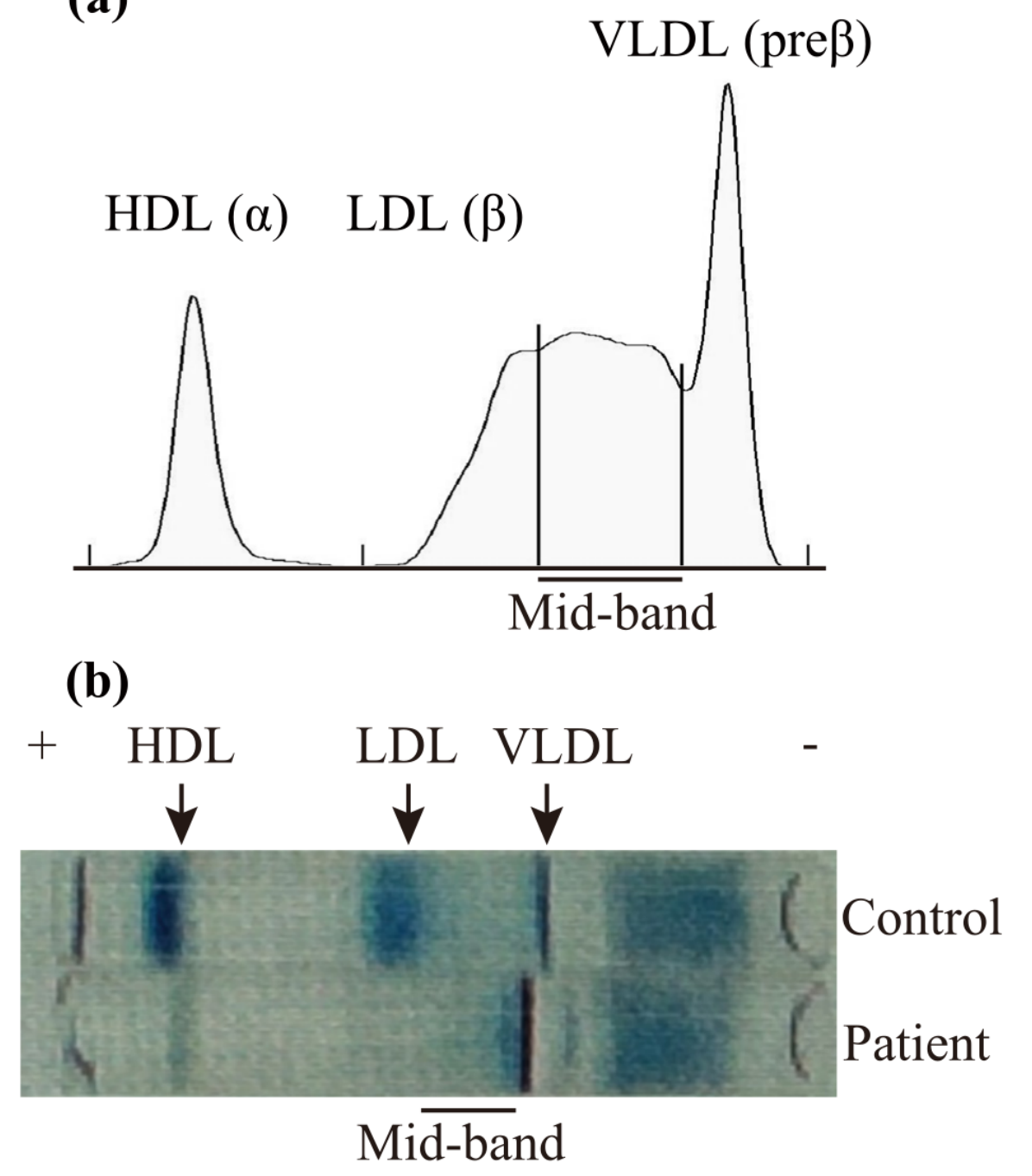

Figure 3 


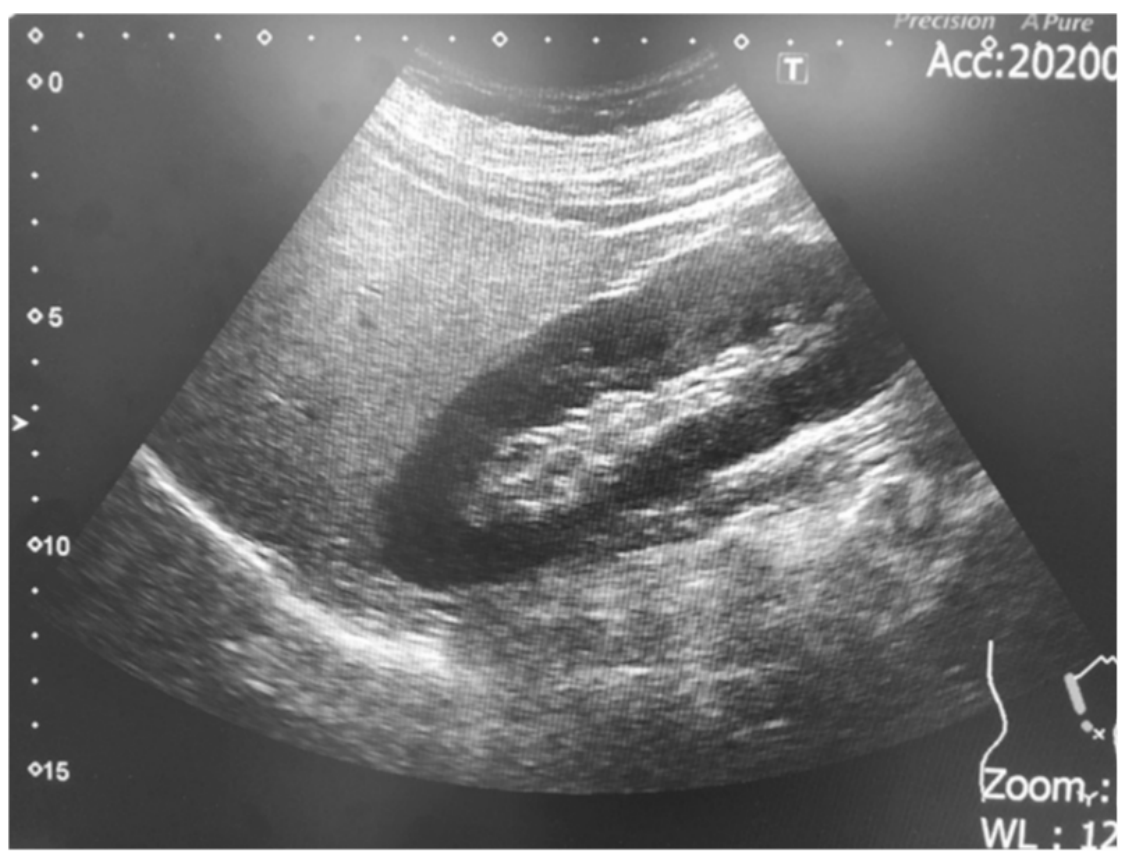

\section{Figure 4}

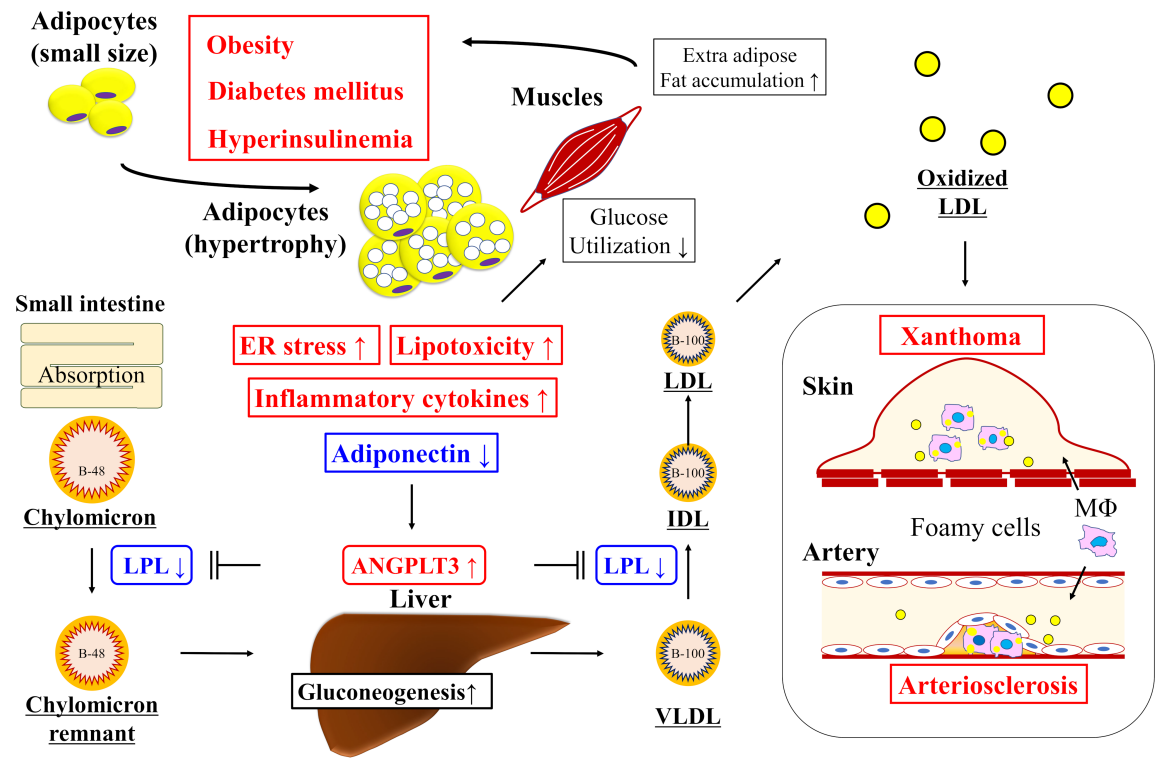

Figure 5 